\title{
Políticas públicas frente a la educación intercultural, de género y contra la violencia
}

\section{Public policies against intercultural, gender and violence education}

\author{
María Caridad Mederos Machado ${ }^{1 *}$, Sandra Josefina Andino ${ }^{1}$ y Margarita Lamas González ${ }^{1}$ \\ ${ }^{1}$ Universidad Estatal Península Santa Elena \\ *mariacamederos@gmail.com
}

DOI: https://doi.org/10.26871/killkana_social.v3i3.517

\begin{abstract}
Resumen
El presente trabajo aborda la problemática de la convivencia libre de violencia por razones de género, interculturales, etc. En el mismo se realiza una revisión sobre las diferentes posiciones que existen referentes al respeto a las diversidades como base para una cultura de paz y convivencia pacífica, así como los elementos que subyacen en la violencia de género. Trata también el papel de las políticas públicas en Santa Elena para la modificación de las causas que generan discriminación. Se emplearon métodos como la revisión documental y la consulta al Plan de Desarrollo y Ordenamiento Territorial (PDOT) de Santa Elena. Se concluye la necesidad de incentivar a todos los niveles y sectores la perspectiva no discriminatoria e inclusiva de desarrollo, que se incluya en las políticas públicas.
\end{abstract}

Palabras clave: Políticas inclusivas, género, interculturalidad y no violencia.

\begin{abstract}
This paper addresses the problem of violence-free coexistence due to gender, intercultural, etc. In it, a review is carried out on the different positions that exist regarding respect for diversity as the basis for a culture of peace and peaceful coexistence, as well as the elements that underlie gender violence. It also deals with the role of public policies in Santa Elena for the modification of the causes that generate discrimination. Methods such as documentary review and consultation of the Development and Land Management Plan (PDOT) of Santa Elena were used. The need to incentivize at all levels and sectors the non-discriminatory and inclusive development perspective, which is included in public policies, is concluded.
\end{abstract}

Keywords: Inclusive policies, gender, interculturality and nonviolence.

\section{Introducción}

Resultan escasos los antecedentes investigativos en la Península de Santa Elena acerca de la temática de respeto a la diversidad y a la convivencia basada en enfoque intercultural. Los elementos de violencia de género que matizan la sociedad peninsular actual son evidencias de la necesidad de una cultura necesaria para la paz familiar y social.

Si bien es cierto que actualmente la presencia de las mujeres en el mercado laboral en el país tiene vital importancia, también se conoce que su contribución al sustento familiar y a la economía del país es altamente valorada. Se reconoce que algunas limitantes que impedían la incorporación de las mujeres, sobre todos negras y mestizas a la vida social, especialmente la laboral, se han ido modificando en los últimos 20 años. Los marcos legales y los niveles de formación alcanzados, así como la escolarización han visibilizado a las mujeres en el mundo laboral.
Según Clemente (2015) La Constitución 2008 (Asamblea Nacional 2009), en sus artículos 340, 341, 342, 347 y 348, avanza hacia el reconocimiento de la participación de las mujeres en la vida pública al proponer entre otros, los siguientes aspectos:

- "El derecho a decidir sobre la vida sexual y reproductiva, así como el reconocimiento de la diversidad de familias".

- "La valoración efectiva del trabajo doméstico no remunerado, la incorporación de la economía del cuidado familiar, la compensación del Estado y la sociedad para las mujeres, el acceso universal a la seguridad social y sus prestaciones incluyendo la jubilación y la renta básica".

- "Medios que garanticen la paridad, alternabilidad y secuencialidad en el sistema electoral ecuatoriano, en los procesos de selección de candidaturas, en las elec- 
ciones y asignación de escaños, en la organización y funcionamiento de los partidos y movimientos políticos, así como la creación de mecanismos de acción positiva para la participación de las mujeres indígenas y afro descendientes".

- $\quad$ "El acceso a la justicia para las mujeres, la imprescriptibilidad de delitos relacionados con violencia de género e intrafamiliar, un sistema de justicia eficiente, ágil, libre de corrupción y que termine con la impunidad. La protección integral de las víctimas de violencia, la reparación y restitución de derechos".

- $\quad$ "El derecho universal de las mujeres a la educación con acciones positivas que permitan erradicar el analfabetismo, el derecho a la educación sexual y a la salud sexual y reproductiva".

Si bien se experimentan avances en las leyes y en el empleo, esto no resulta suficiente para alcanzar la cultura de paz y de convivencia en armonía en el respeto a las diversidades.

El objetivo de este trabajo es realizar una actualización bibliográfica, sobre el tema en cuestión en el Ecuador, y especialmente en Santa Elena.

\section{Desarrollo}

En el Ecuador, según datos de encuestas nacionales, un $60.60 \%$ de mujeres han vivido algún tipo de violencia (física, sicológica, sexual y patrimonial), es decir, que 6 de cada 10 mujeres, independientemente, de su auto identificación, condición social o nivel de educación, ha sufrido algún tipo de violencia. Las mujeres que han vivido maltrato físico, por su cónyuge, según las estadísticas demuestra los porcentajes de violencia intrafamiliar a nivel nacional: violencia física, 68,6\%; y en la provincia de Santa Elena $47,1 \%$ en el Cantón La Libertad $35.5 \%$, en el mismo se encuentra la UPSE. (Campuzano-Catuto, 2015)

Según Salvatierra, la interculturalidad en la formación ciudadana de los estudiantes es un tema que en la actualidad se presenta en las instituciones educativas, como parte principal en el proceso de enseñanza aprendizaje debido a que está ligado como ente fundamental a la búsqueda de una buena convivencia estudiantil y formación, ante situaciones de desintegración por parte de los estudiantes. De acuerdo a una indagación previa realizada en el cantón La Libertad, se pudo conocer que los estudiantes del quinto año presentan problemas de falta de respeto ante la diversidad existente en el entorno educativo, obstaculizando el aprendizaje. (Salvatierra-Lindao, 2016)

Por lo tanto, ante estas problemáticas se necesita entrar en una situación de aprendizaje y educación en cualquiera de los contextos, para modificar las experiencias de violencia e irrespeto a las demás personas, solo por el hecho de considerarles diferentes.

En este sentido las instituciones públicas del territorio, las escuelas y las instituciones de salud, constituyen actores importantes en la modificación de conductas violentas y en fomentar el respeto y las buenas practicas ciudadanas.
Los proyectos que responden a las políticas públicas resultan de suma importancia porque de los mismos se podrá conseguir un modelo de actuación socioeducativa para paliar la situación de violencia social e intrafamiliar, por razones de género y otras, en la provincia de Santa Elena.

En la Constitución de 2008 (arts. 26 y 28) se estableció que la educación es un área prioritaria de la política pública y de la inversión estatal, que responde al beneficio de la ciudadanía y no al servicio de intereses individuales o corporativos; además, se promulgó la Ley Orgánica de Educación Intercultural (LOEI) como norma, tanto para instaurar las regulaciones básicas que permitieron el funcionamiento del Sistema Nacional de Educación, como para profundizar en los derechos y obligaciones de sus actores.

De igual modo intenta potenciar las capacidades de las instituciones dentro de un ambiente de respeto a la interculturalidad, la plurinacionalidad y las identidades diversas, que atendidas desde la vida socio política, educativa y de salud, constituyen fortalezas en la península de Santa Elena.

Las políticas públicas permiten articular acciones en el ámbito social encaminadas a eliminar la discriminación por razones de sexo, orientación sexual, identidad de género, afiliación política, modo de vida, credo, edad, discapacidad, origen nacional o étnico, situación migratoria, condición de salud, entre otros.

Resulta significativo que las políticas, ofrezcan respuesta al Eje 1: Derechos para todos durante toda la vida., contenidos en la Planificación Nacional, mediante el cumplimiento de los Objetivos 1 y 2, referidos a:

1) Garantizar una vida digna con iguales oportunidades para todas las personas.

2) Articular la interculturalidad y plurinacionalidad, revalorizando las identidades.

Los espacios de participación abiertos para consulta y trabajo cotidiano, desde diferentes Planes de Desarrollo Territorial y Nacional demuestran el interés y la necesidad de trabajar desde lo público, los orígenes, la prevención y la atención socioeducativa a la violencia intrafamiliar, como causa primigenia de la violencia social.

Uno de los requerimientos de la población consignados en el Plan Toda una vida es el que se refiere a combatir la "Violencia intrafamiliar y eliminación del feminicidio". De modo que las instituciones públicas deben contar con protocolos y planes de acción concretos para cumplir este rol social. La mayoría de ellas no los ha elaborado, por no tener capacitación suficiente en ese sentido. Las autoras de este documento, se han podido percatar que algunas de las que cuentan con instrumentos para ello, tienen un enfoque asistencial y no preventivo de situaciones de violencia.

Según la Organización de las Naciones Unidas ONU (2016), una de cada tres mujeres son víctimas de violencia intrafamiliar, en sus modalidades de violencia física o psicológica y el Ecuador, no está exento de esa problemática.

Ecuador es uno de los países que presenta mayor cantidad de casos de violencia de género, denunciados en 
el ámbito judicial. Inician con violencia intrafamiliar y el $35 \%$ de ellos termina en feminicidios. Estudios demuestran que el $90 \%$ víctimas mujeres, no proceden a la separación de la pareja, donde la mayoría (44\%) no desea separarse y sólo el $10 \%$ lo concibe como la mejor opción. Pág.9

Las provincias que muestran una mayor violencia de género son: Morona Santiago, Tungurahua, Pichincha, Pastaza y Azuay. Aunque Santa Elena se encuentra entre las provincias con menor índice de violencia contra la mujer, datos proporcionados por el Ministerio del Interior, (Andrade Terán, 2017), ocho de cada diez mujeres divorciadas han sufrido violencia por parte de su pareja, se ha podido incrementar leyes que permiten prevenir y sancionar, casos de violencia contra las mujeres. El $53 \%$ de mujeres en el Ecuador, han denunciado violencia física o sexual de sus parejas. INEC. Pag.3.

En este territorio, la violencia intrafamiliar no solo se basa en la agresión a mujeres por parte de su pareja, sino que existe un $5 \%$ de casos registrados de violencia intrafamiliar por parte de hermanos hacia hermanos, un $7 \%$ de hijos hacia sus padres, un $9 \%$ de suegras y suegros hacia sus nueras y un $79 \%$ de hombres hacia mujeres, dejando así plasmado los tipos de violencia que sufre la sociedad, en el contexto de la familia.

Atendiendo a que las actitudes violentas son aprendidas y no innatas, es que se debe plantear la investigación acción, para llevarla a cabo en los ámbitos de la política pública y por sinergia con las familias de los escolares que están matriculados en las escuelas santaelenenses.

De modo que resulta imperativo trabajar con funcionarios y directivos públicos, con docentes, madres y padres de familia y con personal de instituciones de la salud, por las implicaciones que este tema tiene en la salud sexual y reproductiva y en la salud general de las personas.

\section{El respeto a las diversidades como base para una cultura de paz y convivencia pacífic}

a

La diversidad es intrínseca al desarrollo humano y social. Las formaciones económicas sociales han diferenciado a los seres humanos, desde sus orígenes en relación a diferentes criterios, pero los más fuertes y demarcados en los últimos tiempos se relacionan con las prácticas religiosas, la cultura y el género.

Se suceden en la ciencia y en el concepto popular, muchas interpretaciones y posturas en relación con este fenómeno, que algunas de ellas, especialmente las populares, el irrespeto a quien no es mayoría o no cumple "la norma", referida a términos estadísticos. También la distancia de la posición social hegemónica está marcando los niveles de respeto en la escala ciudadana.

En lo cultural, es esencial la superación de posturas unívocas para dar paso a formas plurales de comprensión, pues de ellas se deduce que no hay una sola verdad, sino que encontramos realidades construidas sobre el intercambio de ideas, el reconocimiento mutuo y el consenso (Foucault y del Camino, 1977; Weir, 2008); se puede pensar tanto en la descolonización del Estado y las mentes, como en la superación del dominio cultural (Quijano, 2001; Walsh, 2009).

La educación es fin y es medio para alcanzar un cambio cultural y desterrar prejuicios y estereotipos que durante siglos han sustentado la marginación de las diversidades, han atentado contra la identidad cultural y al mismo tiempo han desarrollado la idea postmodernista de que se es lo mismo en términos culturales, aun cuando las historias de vida hayan tenido lugar en espacios físicos, naturales, culturales, y familiares diferentes.

Los contextos comunitarios son espacios de formación, que conducidos con ideas claras del concepto pedagógico y de la equidad, practicada en las leyes y en las políticas públicas, conducen también al cambio cultural, que transforma los prejuicios y estereotipos en ideas y criterios no discriminatorios y flexibles sobre el respeto a la diferencia.

El Plan Nacional de Desarrollo 2017-2021, Toda una vida, reconoce que la cohesión territorial es entendida como justicia social y territorial. En el sentido de la garantía de derechos, se enmarca en la protección integral durante el ciclo de vida, proceso alineado a la lucha contra la desigualdad, al fortalecimiento de la participación y corresponsabilidad ciudadana, y al compromiso del Estado de proveer aquellos bienes superiores que representan derechos ciudadanos. Desde la perspectiva territorial considera como ejes fundamentales la sustentabilidad ambiental y la gestión de riesgos.

La cohesión territorial busca reducir las brechas, ampliar las oportunidades y mejorar las condiciones de habitabilidad, así como, erradicar la pobreza extrema y mejorar la calidad de vida. Ello demanda actuar de manera efectiva en el mejoramiento de la prestación de servicios vinculados a la reproducción social y la satisfacción de necesidades básicas en materia de hábitat y vivienda, reduciendo las inequidades entre zonas urbanas y rurales.

$\mathrm{Si}$ bien estos elementos que impulsa el estado se encuentran en la base de la planificación del desarrollo, los elementos psico socio educativos que acompañan esas decisiones, deben estar guiados por buenas prácticas de convivencia y articularse entre las diferentes instituciones en las que el individuo se desarrolla desde su nacimiento.

Es importante que se consideren en estas acciones las encaminadas a legitimar la cultura científica y en el respeto que merece la cultura popular, desmitificar las razones y prejuicios de segregación y discriminación que muchas veces subyace en las creencias y estereotipos de la cultura popular. La cultura científica, de acuerdo a Olivé (2011), se refiere por una parte al conjunto de prácticas, representaciones, normas y valores dentro de una comunidad científica. Por otra parte, la cultura de un grupo social que no es científico se refiere al conjunto de representaciones y valores que provienen de la ciencia y que de alguna forma 
se ha incorporado en las representaciones y prácticas de dicho grupo social.

\section{Violencia de género}

Según Mañas Viejo (2017) la interiorización intersubjetiva de la desigualdad se manifiesta en la pervivencia de los estereotipos más simples y de forma profunda, tanto que no dejan paso a las evidencias y se basan, aún en personas con cierta intelectualidad reconocida, en argumentos basados en creencias y opiniones

La autora concluye que la violencia social es percibida como un gran problema social por la población en general y que no existen diferencias notables, aunque las mujeres con mayor frecuencia, perciben la desigualdad de género y por tanto la violencia ejercida sobre ellas por el hecho de ser mujer.

En el estudio de Mañas Viejo (2017), los hombres, son los están mejor formados y tienen un empleo más estable, quienes con más frecuencia perciben la desigualdad. Estos hechos ponen en evidencia la importancia de la formación, del acceso a la educación, como eje de intervención política en el avance de una sociedad cada vez más equitativa.

Papel de la sociedad y la educación en la modificación del modelo socioeducativo

Por otro lado, se considera la coeducación, entendida como un modelo psicopedagógico que integra y valora por igual las contribuciones de ellos y de ellas, no sólo al conocimiento científico, también a la experiencia vital es una herramienta a desarrollar y extender en el aula más allá del aula.

Aproximadamente el $40 \%$ de los docentes, que perciben el tema de la desigualdad de género manifiesta no tener acceso a formación ni recursos para enfrentarlo. La autora concluye que la percepción de la violencia de género se percibe como fuente de desigualdad por toda la sociedad, sin embargo, las diferencias existentes sobre esta percepción entre hombres y mujeres apuntan la dirección del trabajo a realizar en un futuro.

Las instituciones educativas y de salud, cumplen una función importante como actores del cambio social y cultural. Por la implicación que tienen ambos espacios en la vida humana, es necesario impulsar la investigación acción y los laboratorios de investigación, aunque la misma se desarrolle en los escenarios naturales de la vida cotidiana. Las modelaciones conductuales y sus análisis son una forma de eliminar sesgos cognitivos en los comportamientos y modificaciones de las teorías implícitas de los participantes.

\section{Conclusiones}

Las políticas públicas y su seguimiento constituyen el punto de partida, para la comprensión y modificación de las causas que generan y perpetúan las desigualdades sociales, conducentes a relaciones de poder, donde se desata la violencia de toda índole, con supremacía de la intrafamiliar.
Las instituciones educativas de todos los niveles, las de atención primaria de salud, las familias y los medios de comunicación son actores importantes en la transformación de las actitudes que promueven y practican la violencia. Con políticas públicas intencionadas a ese objetivo, los indicadores pueden ser cambiados y transformados hacia mejores augurios en las relaciones interpersonales y en la convivencia social.

\section{Referencias Bibliográficas}

Andrade Terán, A. A. (2017). Valoración cuantitativa y cualitativa de casos de violencia intrafamiliar registrados en el cantón La Libertad, Provincia de Santa Elena. (Tesis Doctoral, Universidad Católica de Santiago de Guayaquil). Descargado de http: / / repositorio.ucsg.edu.ec/ handle/3317/8224

Campuzano-Catuto, L. V. (2015). Empleo y trabajo rural en Santa Elena: caso comuna Loma Alta parroquia Colonche 2015 (B.S. thesis). La Libertad: Universidad Estatal Península de Santa Elena, 2015.

Clemente Borbor, M. E. (2015). Los factores jurídicos como principio del derecho en el Delito de violencia física contra la mujer. Un estudio comparativo en la ciudadela general Enríquez Gallo, La Libertad. 2014 (Tesis Doctoral, La Libertad: Universidad Estatal Península de Santa Elena, 2015). Descargado de https://repositorio.upse . edu.ec/xmlui/handle/46000/3803

COIP - Código Orgánico Integral Penal Del Ecuador. (2013, Feb). Descargado de https:// www.scribd.com/doc/192099212/ COIP-Codigo-Organico-Integral-Penal -Del-Ecuador

Consejo Nacional de Planificación. (2017). Plan Nacional de Desarrollo 2017-2021-Toda una Vida. Ecuador.

Foucault, M., y del Camino, A. G. (1977). La arqueología del saber. Siglo XXI.

Mañas-Viejo, C., Molines Alcaraz, M., Martínez Sanz, A., Cerdá, E., del Mar, M., Montesinos Sánchez, N., ... others (2017). Percepción de la violencia de género y necesidad de cambio cognitivo.

Quijano, A. (2001). Colonialidad del poder, globalización y democracia. Utopías, nuestra bandera: revista de debate político(188), 97-123.

Ramírez, L. L., Romero, X. R., Peinado, E. S., Franco, A. G., García, J. C., y Feltrero, R. (2017). Educación, comunicación y apropiación de la ciencia desde una perspectiva pluralista: experiencias en la construcción del diálogo para la apropiación social de los conocimientos. Revista Iberoamericana de Ciencia, Tecnología y Sociedad-CTS, 13(38), 205-226.

Salvatierra-Lindao, D. D. (2016). La interculturalidad en la formación ciudadana de los estudiantes de quinto grado del centro de educación general básica once de diciembre del cantón la libertad, provincia de 
santa elena, periodo lectivo 2015-2016. (B.S. thesis).

La Libertad: Universidad Estatal Península de Santa Elena, 2016.

Walsh, C. E. (2009). Interculturalidad, estado, sociedad: luchas (de) coloniales de nuestra época. Universidad Andina Simón Bolívar.

Weir, L. (2008). The concept of truth regime. Canadian Journal of Sociology, 33(2).

Recibido: 16 de agosto de 2019

Aceptado: 25 de noviembre de 2019 
